\title{
Free BDD based CAD of Compact Memristor Crossbars for in-Memory Computing
}

\author{
Amad Ul Hassen \\ University of Central Florida, USA \\ amad@eecs.ucf.edu \\ Haseeb Aslam Butt \\ KTH Royal Institute of Technology, Sweden \\ habutt@kth.se
}

\author{
Salman Anwar Khokhar \\ University of Central Florida, USA \\ skhokhar@eecs.ucf.edu \\ Sumit Kumar Jha \\ University of Central Florida, USA \\ jha@eecs.ucf.edu
}

\begin{abstract}
The demise of Moore's law, breakdown of Dennard Scaling, dark silicon phenomenon, process variation, leakage currents and quantum tunneling are some of the hurdles faced in the further advancement of computing systems today. As a result, there is a renewed interest in alternate computing paradigms using emerging nanoelectronic devices. This work uses free binary decision diagrams (FBDDs) for computer-aided design (CAD) of compact memristive crossbars for sneak-path based in-memory computing. The absence of a fixed variable ordering makes FBDDs more compact than their ordered counterpart called reduced ordered binary decision diagrams (ROBDDs). Our design has used the size of the circuit-representation of Boolean functions for selecting different variable orderings along different paths which results in compact FBDDs. We have demonstrated our approach by designing compact crossbars for a four-bit multiplier and other RevLib benchmarks. Our synthesis process yields a $50.1 \%$ reduction in area over the previous FBDD-based synthesis for the fourth-output-bit of the multiplier. Overall, our approach has reduced the multiplier area by $20.1 \%$.
\end{abstract}

\section{CCS CONCEPTS}

- Hardware $\rightarrow$ Emerging technologies; Emerging architectures; Electronic Design Automation; Emerging tools and methodologies; - Computer Systems Organization $\rightarrow$ Architecture;

\section{KEYWORDS}

memristor, crossbar, binary decision diagrams, BDD, boolean functions, synthesis of crossbars, in-Memory computing.

ACM Reference Format:

Amad Ul Hassen, Salman Anwar Khokhar, Haseeb Aslam Butt, and Sumit Kumar Jha. 2018. Free BDD based CAD of Compact Memristor Crossbars for in-Memory Computing. In Proceedings of IEEE/ACM International Symposium on Nanoscale Architectures (NANOARCH '18). ACM, New York, NY, USA, 7 pages. https://doi.org/10.1145/3232195.3232222

Permission to make digital or hard copies of all or part of this work for personal or classroom use is granted without fee provided that copies are not made or distributed for profit or commercial advantage and that copies bear this notice and the full citation on the first page. Copyrights for components of this work owned by others than ACM must be honored. Abstracting with credit is permitted. To copy otherwise, or republish, to post on servers or to redistribute to lists, requires prior specific permission and/or a fee. Request permissions from permissions@acm.org.

NANOARCH '18, Fuly 17-19, 2018, Athens, Greece

(C) 2018 Association for Computing Machinery.

ACM ISBN 978-1-4503-5815-6/18/07 .. \$15.00

https://doi.org/10.1145/3232195.3232222

\section{INTRODUCTION}

The stellar growth in Moore's law was sufficient to maintain the steady growth in computing capabilities defying the shortcomings of the Von-Neumann architecture [1]. Separate physical locations for data-processing and data-storage have made bus-speed the main bottleneck in the classical Von-Neumann computing architecture [2]. Integrated circuits have already packed so many transistors that all of them cannot be powered up at the same time without violating temperature constraints, a phenomenon characterized as dark-silicon. Limited bus-speed, dark silicon and Dennard scaling have reduced the gains from further miniaturization of CMOS devices for general purpose computing architectures [3]. These challenges cannot be remedied without fundamental changes in current computing architectures. This has renewed interest in innovative low-cost computing alternates employing emerging nanoelectronic devices [4]. One such recent computing paradigm is in-memory computing, where computing and storage are performed alongside each other [5]. Our work uses memristor as a basic element for in-memory computing. The resistance of a memristor varies with the amount and the direction of the current flowing through it. In the absence of flow of current, the memristor retains its resistance and acts as a non-volatile memory. A memristor with the minimum resistance $\left(R_{O N}\right)$ is said to be in ON state, while a memristor with the maximum resistance $\left(R_{O F F}\right)$ is said to be in OFF state. Depending upon memristive technology, the ratio of $R_{O F F}$ to $R_{O N}$ can vary from a few hundreds to $10^{7}[6]$. When the ratio $\frac{R_{O F F}}{R_{O N}}$ is high, a memristor can serve as a two-terminal switch.

Ever since the discovery of the first memristor in 2008 [7], computing with memristive circuits has taken two disparate paths. The first approach implements a material-IMPLY circuit from two memristors and uses it as a basic unit for constructing memristive circuits [8] [9] [10]. The second approach uses memristor as a two-terminal switch. Our work falls under the second category and uses memristor as a two-terminal switch for in-memory computing on crossbars. Shannon's Master's thesis was the first comprehensive study on implementation of Boolean functions using two-terminal switches called relays [11]. Figure 1a shows a circuit for computing of the simple Boolean function $f=\neg A \neg B+A B C D$ using two-terminal switches. This circuit will become complete allowing the current to flow through the sense resistor $R_{S}$ whenever either of the following two conditions is fulfilled, (1) Both A and B are false (2) All four inputs $\mathrm{A}, \mathrm{B}, \mathrm{C}, \mathrm{D}$ are true.

Flow or sneak-path based in-memory computing uses the above concept for computing Boolean functions on crossbars; where each 
cross-point memristor mimics the behaviour of a two-terminal switch. Depending on input values, the memristors of a crossbar are configured either in ON or OFF state. For sneak-path based in-memory computing on a crossbar, a series of interconnected ON memristors forms a low resistance flow-path between the input and the output nanowires whenever the target function $f$ is true. Figure $1 \mathrm{~b}$ shows an implementation of the above mentioned Boolean function $f$ on a $3 \times 3$ crossbar. This crossbar allows a sneak-path between the bottom and the topmost nanowires when either of the above-mentioned two conditions is fulfilled. For example, if A is false, the current injected in the bottom nanowire will reach the first column through the memristor labelled as $\neg A$, if $\mathrm{B}$ is also $f$ alse, the memristor labelled as $\neg B$ will connect the first column with the topmost nanowire; thus completing a sneak path between the bottom and the topmost nanowires. This sneak path is highlighted by the blue line in figure 1b. Similarly, when all four inputs A, B, $\mathrm{C}$ and $\mathrm{D}$ are true, the corresponding memristors in figure $1 \mathrm{~b}$ will be in ON state, thus creating a low resistance sneak-path which is highlighted by the red line in figure $1 \mathrm{~b}$. One can observe oneto-one correspondence between the flow-paths of figure 1a and sneak-paths of figure $1 \mathrm{~b}$.

\section{RELATED WORK}

Kvatinsky et al. [12] proposed Memristor-Aided Logic (MAGIC) in 2014 for implementing Boolean functions on memristive circuits. They used MAGIC for designing simple NAND and NOR gates using ideas described in Shannon's seminal work on switching circuits [11]. Jha et al. introduced flow-based computing for implementing Boolean functions on memristive crossbars [13]. Their synthesis process transformed Boolean functions into negation normal (NNF) before mapping it onto a crossbar. They gave two simple rules for structural implementation of the NNF of a function on a crossbar. They proved that their flow-based computing can evaluate any Boolean function on a sufficiently large memristive crossbar. In contrast to other approaches requiring a customized fabrication of memristive circuits, the flow-based computing aimed at implementing Boolean logic on pre-fabricated memristive crossbars, thus benefiting from economy-of-scale of easily manufactured application independent generic crossbars. Theoretically, their method guarantees that any Boolean formula can be computed on memristive crossbars, but it requires prohibitively large crossbars because the NNF of a function can result in exponentially sized representation. In their later work, Velasquez et al. designed compact crossbars using formal methods [14]. They demonstrated their approach by synthesizing a compact 1-bit adder, but this approach also could not scale up for complex functions because its computational complexity was doubly exponential in terms of crossbar size. Finally Hassen \& Chakraborty et al. [15] [16] employed reduced ordered binary decision diagrams (ROBDDs) for synthesizing crossbars for in-memory computing of arbitrary Boolean functions. In contrast to previous approaches lacking scalability, the ROBDD based synthesis is capable of capitalizing on the benefits of economy-of-scale of application independent physical crossbars. Later, Hassen et al. [17] proposed the use of free binary decision diagrams (FBDDs) to increase the area efficiency of the synthesized crossbars. FBDDs have more representational power than their ordered counterpart, thus

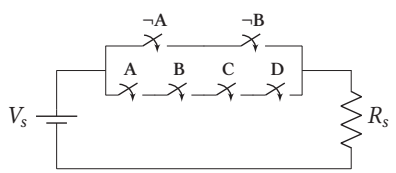

(a)

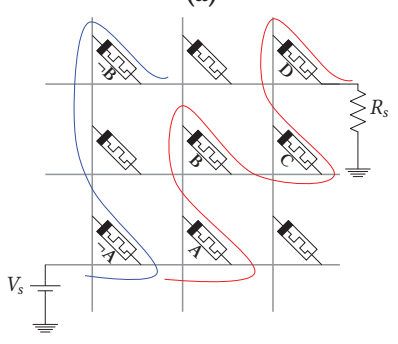

(b)

Figure 1: (a) Switch based circuit for computing the Boolean formula $f=\neg A \neg B+A B C D$ using the flow of electric current. (b) Crossbar for sneak-path based or flow-based in-memory computing of $f$, blue and red lines highlight the sneak-paths responsible for computing $f$.

they can represent a Boolean function more compactly than ROBDDs. Hassen et al. used greedy heuristics on the disjunctive normal form (DNF) of a Boolean function for synthesizing its crossbar.

This work also employs FBDDs for synthesizing crossbars; the novelty of our approach lies in the use of the size of circuit representations of Boolean functions for adjusting variable orderings along different paths of FBDDs, which result in compact crossbars. Our approach has produced $20.1 \%$ more compact 4-bit multiplier than the previous FBDD-based synthesis [17]. It has also reduced the crossbar size for the fourth-output-bit of the multiplier from $(35 \times 34)$ to $(24 \times 24)$; making it $50.1 \%$ more compact than the previous design approach [17]. The rest of the paper is organized as follows: first, we give the necessary background on sneak-pathbased/flow-based computing, BDDs, FBDDs, advantages of FBDDs over ROBDDs; then we describe our approach for synthesizing memristive crossbars using compact FBDDs. We compare our approach with the previous FBDD-based synthesis for multiplier and other benchmarks in the experimental section. Finally, we conclude the paper with an overview of our results.

\section{BACKGROUND}

\subsection{In-Memory Computing using Sneak-Paths}

A crossbar consists of arrays of horizontal and vertical nanowires. Each vertical nanowire of a crossbar is connected with all other horizontal nanowires through separate memristors and vice versa. Cross-point architecture of crossbar intertwines data-storage and data-processing to facilitate flow-based in-memory computing. The state of individual memristors stores inputs in binary form. The cross-point architecture, resistance of individual memristors as well as their position and connections with the nanowires are responsible for flow-based computing.

Crossbar nanowires are made up of conductive material; therefore, each nanowire can be considered a node. Thus, an $n \times m$ 
crossbar has $n+m$ nodes and $n m$ memristive connections between the nodes or nanowires. For a large crossbar, the number of memristive connections is disproportionately more than the number of nanowires, which gives rise to the phenomenon of sneak-paths. A sneak-path is a low-resistance-path between any two nanowires which are not connected with each other through an ON memristor Sneak-paths are responsible for faulty reads when crossbars are used for storage purpose. The number of sneak-paths increases exponentially with their length [18]. Although, this profusion of sneak-paths makes them a nuisance for storage related applications, in-memory computing leverages their very abundance to implement Boolean functions. The objective of sneak-path based synthesis is to manage the sneak-paths between the input and the output nanowires such that the current injected into the input nanowire flows out of the output nanowire only if the target function is true. Consequently, such sneak-path based computing is also known as flow-based in-memory computing [13] [16] [15] [14]. In the context of crossbars, flow-based in-memory computing and sneak-path-based in-memory computing are synonymous. The task of designing crossbars for sneak-path-based or flow-based in-memory computing is defined as follows [16],

Definition 1 (SNeak-Paths based Synthesis of Crossbars). Let $f:\{0,1\}^{k} \rightarrow\{0,1\}$ be a Boolean function with $k$ inputs $I=\left\{b_{1}, b_{2}, b_{3} \ldots b_{k}\right\}$, the objective is to map these $k$ inputs or their complements on crossbar memristors such that the following two conditions are fulfilled for any input $x \in\{0,1\}^{k}$

- If $f(x)=1$, then there exists a sneak-path between the input and the output nanowires.

- If $f(x)=0$, then there is no sneak-path between the input and the output nanowires.

In our design, we have designated the bottom nanowire as input nanowire, while the top nanowire serves as output. Figure $1 \mathrm{~b}$ shows additional circuitry for testing the existence of a sneak-path. A small sense voltage $V_{s}$ injects current into the bottom nanowire, a sense resistor $R_{S}$ detects the amount of current flowing out of the topmost nanowire. When the function is true, low resistance of sneak-paths allow a significant amount of current to flow out of the topmost nanowire, resulting in significant voltage drop across the sense resistor $R_{s}$. When the target function is false, very little current flows out of the topmost nanowire, resulting in significantly smaller voltage drop across $R_{S}$.

\subsection{Binary Decision Diagrams}

Binary decision diagrams (BDDs) are compact graphical representation of Boolean functions. Lee used them for modeling switching circuits in 1959 [19]. Akers et al. used them for implementing digital circuits [20]. Let $f(x)$ be a k-bit function on the variable set $\mathrm{V}=$ $\left\{v_{1}, v_{2}, v_{3} \ldots v_{k}\right\}$, then its BDD is a rooted directed graph with two terminal nodes and several non-terminal nodes. All non-terminal nodes of a BDD are labeled as $v_{i} \in V$, while the terminal nodes are labeled as 0 or 1 . By definition of a BDD, if the function $f(x)$ is true for some $x \in\{0,1\}^{k}$ then there exists a path from the root node to the terminal node labeled ' 1 '; the path reaches the terminal node labeled ' 0 ' for a false valuation of $f(x)$. Reduced ordered binary decision diagrams (ROBDDs) are the most popular among all BDDs.
Randal Bryant proved the canonicity of ROBDDs for a globally fixed variable ordering [21]. This property led to the discovery of efficient algorithms for their synthesis. Let $\pi=\left\{v_{1}, v_{2}, v_{3} \ldots v_{k}\right\}$ be a variable ordering for an ROBDD, then $v_{i}$ always appears before $v_{j}$ for all $i<j$ on any path from the root node to the terminal nodes; such an ROBDD is called $\pi$-ROBDD. Depending upon the nature of original function, the size of an ROBDD can vary drastically for different variable orderings. For a bad variable ordering, the ROBDD can become exponentially large [22]. Finding an optimal variable ordering for an ROBDD is an NP-hard problem; although there are several advanced heuristics which can find reasonably good variable orderings during the construction of an ROBDD.

In contrast to ROBDDs, the requirement of fixed variable ordering is not forced on free binary decision diagrams (FBDDs). Variables are allowed to appear in different orderings on different paths between the root node and the terminal nodes, thus ROBDDs are a special case of FBDDs. The freedom to have different variable orderings along different paths gives FBDDs tremendous representational power. There are several functions with exponentially large ROBDDs and polynomially sized FBDDs [22]. We exploit this increased representational power of FBDDs for synthesizing compact crossbars.

\section{PROPOSED DESIGN}

Our sneak-path-based synthesis maps input variables on crossbar memristors such that the conditions specified in Definition 1 are fulfilled. The mapped variables control the state of the target memristors such that a sequence of inter-connected $\mathrm{ON}$ memristors creates a low resistance sneak-path between the bottom and the topmost nanowires whenever the target function $f(x)$ is true, and there is no sneak-path between them otherwise. This situation is similar to BDDs where a sequence of ON edges creates a flow-path between the root node and the terminal node labeled ' 1 ' for a true valuation of the target function $f(x)$. The resemblance of flow-paths of BDDs and sneak-paths in crossbars makes BDDs a natural choice for synthesizing the latter.

Despite the popularity of ROBDDs, we are using FBDDs in our synthesis process. FBDDs have no requirement of a globally fixed variable ordering; thus, they allow variables to appear in different orders along different paths in order to achieve structural optimization both on global and local levels. This freedom of variable ordering opens up a new quest: how to choose a particular variable ordering along a given path between the root node and the terminal nodes? Most of the work on ordering variables has revolved around ROBDDs. Our previous work used greedy heuristic on the disjunctive normal form (DNF) of Boolean formulae for selecting different variable orderings along different paths of an FBDD [17]. This work has improved FBDD-based synthesis further by using the size of circuit representation of Boolean functions as the main criterion for searching favorable variable orderings along different paths of the FBDD.

Besides variable ordering criterion, the remaining steps are similar to our previous work, as shown in figure 3 [17]. The synthesis starts with the simplification of the Disjunctive Normal Form (DNF) of the target function $f(x)$. In the second step, we synthesize an FBDD for $f(x)$ using Shannon expansion at each node. The choice 


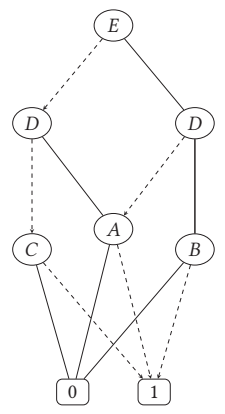

(a) FDDD

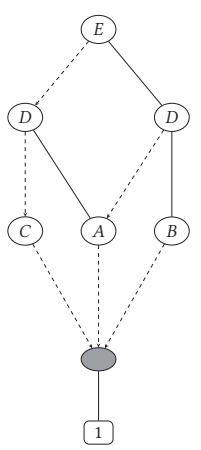

(b) Pruned bipartite FDDD
Figure 2: (a) FDDD for alu_9 benchmark synthesized using our approach. (b) Pruned bipartite FDDD for alu_9.

of variables for Shannon expansion plays a critical role in determining the size of FBDD. Each node is decomposed around that binary variable which results in the smallest sized circuit representations for the functions represented by its children. The next section elaborates the details of our variable selection criterion. Figure 2a shows the FBDD of alu_9 benchmark designed using our approach. The third step is pruning of the FBDD, which removes edges connected with the terminal node labeled ' 0 '. These edges are extraneous because paths ending on the terminal node labeled ' 0 ' are not relevant to flow-based computing. The fourth step converts the pruned FBDD into a bipartite graph. This step is needed because crossbar can only be modeled by a bipartite graph. We transform the pruned FBDD into a bipartite graph by inserting dummy nodes into odd length cycles. Figure $2 \mathrm{~b}$ shows the the pruned bipartite graph for alu_9 benchmark. Finally we map the bipartite version of the pruned FBDD onto a crossbar and verify its functionality. For mapping, distance of each node is measured from the root node. The root node is mapped onto the topmost nanowire. Even numbered non-terminal nodes are represented by horizontal nanowires and odd numbered non-terminal nodes are represented by vertical nanowires. Once the graph becomes bipartite, the distance between the root node and any other node would be either an even-number or an odd-number but not both. If the distance of the terminal node from the root node is not an even number, an additional dummy node is inserted in order to enable its mapping on the bottom nanowire. Figure $2 \mathrm{~b}$ highlights the dummy node added due to this reason. Figure 4a shows the final crossbar synthesized for the alu_9 benchmark, while figure 4 b plots output voltages for each combination of inputs. Red bars show voltage levels corresponding to true outputs of alu_9, while blue bars indicate voltage levels for false outputs. Next section describes variable selection criterion which is responsible for synthesizing more compact FBDDs than the previous FBDD-based synthesis [17].

\subsection{Variable Ordering Procedure}

An FBDD can have an exponential number of paths between its root node and the terminal nodes; and each path is allowed to have its own variable ordering. Thus the problem of finding optimal

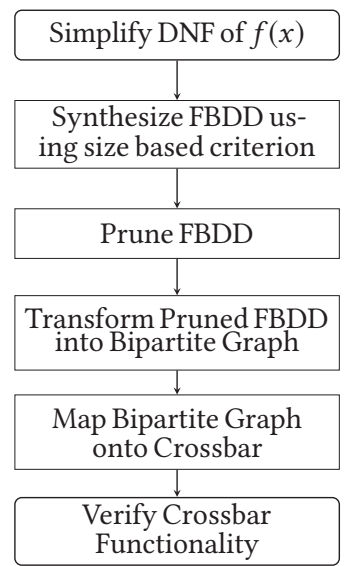

Figure 3: Flow diagram of our FBDD-based synthesis.

variable orderings along all paths of an FBDD can is harder than finding one global variable ordering for an ROBDD. It is important to mention that the problem of finding an optimal variable ordering for an ROBDD is NP-hard [22]. Fortunately, there are several computationally efficient heuristics which can find reasonably good global variable ordering for designing compact ROBBDs. Several ROBDD ordering heuristics use the properties of corresponding circuit representation of Boolean formulae for finding reasonably good global variable orderings [23] [24]. We are using the size of circuit-representation of Boolean formulae represented by non-terminal nodes of an FBDD for ordering variables along different paths between the root node and the terminal nodes. Since each path can have its own variable ordering, our approach repeatedly uses the criterion of equation 1 at each decomposition step during the construction of an FBDD. Let $f(x):\{0,1\}^{k} \rightarrow\{0,1\}$ be a function represented by some non-terminal node $n$ in our FBDD, then the node $n$ is decomposed around the binary variable $v \in x=\left\{b_{1}, b_{2}, b_{3} \ldots b_{k}\right\}$ such that it results in the most compact circuit implementations for the co-factors/functions represented by the children of $n$. More formally,

$$
v=\underset{b_{i} \in\left\{b_{1}, b_{2}, b_{3} \ldots b_{n}\right\}}{\operatorname{argmin}}\left(\operatorname{size}\left(f\left(b_{i}\right)\right)+\operatorname{size}\left(f\left(\neg b_{i}\right)\right)\right)
$$

In the above equation, $v$ is the binary variable used for Shannon decomposition of $f(x)$, size(-) represents the total number of 2-input AND and OR gates in the corresponding circuit implementations of the input function. Estimation of circuit-size from the DNF of a function is a straight forward two-step process. The DNF represents a function as sum of products (SOP). A product term with $i$ literals can be realized using $(i-1) 2$-input AND gates. Similarly, if the DNF has $j$ product terms, then they can be OR-ed together using $(j-1) 2$-input OR gates. Circuit size is calculated as the total number of AND and OR gates obtained in the above two steps. All non-terminal nodes of the FBDD are split around the variables chosen according to the criterion of equation 1. Once FBDD is constructed, the resulting orderings along all paths between the root node and the terminal nodes satisfy the equation 1 . Table 1 illustrates this process of choosing a binary variable $v$ for the decomposition of the root node of alu_9 benchmark. In this table, $f$ is 
Table 1: Illustration of variable-selection-procedure at the root node of alu_9 benchmark, the root node implements $f(A, B, C, D, E)=\neg B D E+\neg A \neg D E+\neg A D \neg E+\neg C \neg D \neg E$

\begin{tabular}{cccccc}
\hline $\mathrm{v}$ & $f_{0}=f(v=0)$ & $\operatorname{size}\left(f_{0}\right)$ & $f_{1}=f(v=1)$ & $\operatorname{size}\left(f_{1}\right)$ & $\operatorname{size}\left(f_{0}\right)+\operatorname{size}\left(f_{1}\right)$ \\
\hline$A$ & $\neg B D E+\neg D E+D \neg E+\neg C \neg D \neg E$ & 9 & $\neg B D E+\neg C \neg D \neg E$ & 5 & 14 \\
$B$ & $D E+\neg A \neg D E+\neg A D \neg E+\neg C \neg D \neg E$ & 10 & $\neg A \neg D E+\neg A D \neg E+\neg C \neg D \neg E$ & 8 & 18 \\
$C$ & $\neg B D E+\neg A \neg D E+\neg A D \neg E+\neg D \neg E$ & 10 & $\neg B D E+\neg A \neg D E+\neg A D \neg E$ & 8 & 18 \\
$D$ & $\neg A E+\neg C \neg E$ & 3 & $\neg B E+\neg A \neg E$ & 3 & 6 \\
$E$ & $\neg A D+\neg C \neg D$ & 3 & $\neg B D+\neg A \neg D$ & 3 & 6 \\
\hline $\operatorname{argmin}\left(\operatorname{size}\left(f_{0}\right)+\operatorname{size}\left(f_{1}\right)\right)=\mathrm{E}$ & & & & $\min \left(\operatorname{size}\left(f_{0}\right)+\operatorname{size}\left(f_{1}\right)\right)=6$ \\
\hline
\end{tabular}

the Boolean function representing the alu_9 benchmark. The first column lists all five binary variables of $f$, the second and the fourth columns list the DNF representations of the co-factors of $f$, the third and the fifth columns are the sizes of circuit-representations of the co-factors, while the last column lists the total size of circuit representations of both co-factors of $f$. Both $\mathrm{D}$ and $\mathrm{E}$ result in the smallest circuit implementations of the co-factors of $f$. Figure 2a shows the corresponding FBDD for alu_9 where the root node is resolved around the binary variable $\mathrm{E}$.

\section{EXPERIMENTAL RESULTS}

We have synthesized crossbars for all eight output-bits of a 4-bit multiplier using our approach. The reason for choosing the multiplier to demonstrate our approach is the variation in its complexity; the complexity of its output-bits increases from the least-significantbit (LSB) to the middle-output-bit, then it starts decreasing till the most-significant-bit (MSB). We have synthesized a separate crossbar for each of the eight output-bits of the multiplier. Table 2 gives the sizes of multiplier crossbars designed using our approach. The effects of increasing complexity are evident by the larger size requirements of the crossbars. For comparison, we have also listed the sizes of the crossbars designed using previous FBDD-based approach [17]. Our approach has reduced the size of the crossbar for middle-output-bit by $50.1 \%$. Overall, our approach has resulted in $20.1 \%$ reduction in the area requirement as compared to the previous approach [17].

We have verified the functionality of each crossbar by applying all input combinations, sneak-path existed only for those inputs for which the function evaluated to true. Figure 5 shows the output of HSPICE simulation for the $24 \times 24$ crossbar for the fourth-outputbit of the multiplier. The X-axis is the index of truth-table entry for input operands, while the Y-axis represents the output voltage across the sense resistor $R_{s}$. Blue bars show voltage levels corresponding to false outputs and red bars correspond to true outputs. For this simulation, we have used $V_{s}=1 \mathrm{~V}, R_{s}=100 \Omega, R_{O N}=$ $100 \Omega$ and $\frac{R_{O F F}}{R_{O N}}=10^{4}$. The output voltage for a false valuation was always less than $35 \mathrm{mV}$, and the voltage for true outputs was always greater than $172 \mathrm{mV}$. The gap of $137 \mathrm{mV}$ is wide enough for error-free sensing of a sneak-path.

We have also used our approach for synthesizing the RevLib benchmarks in table 3 [25]. This table also compares the results with the previous FBDDs-based synthesis employing the most frequent variable as splitting criterion [17].

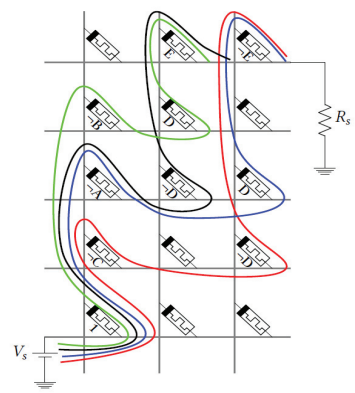

(a)

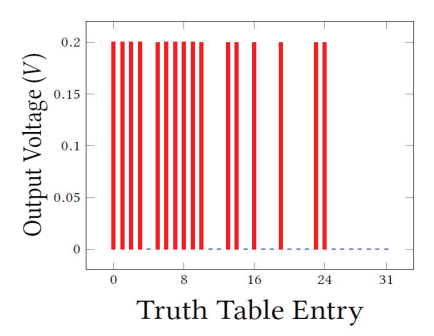

(b)
Figure 4: (a) Crossbar for sneak-path based in-memory computing of alu_9 benchmark. The four sneak-paths are highlighted by red, green, blue and black lines. (b) Bar plot showing the voltage of the topmost nanowire. The truth table entries are depicted at the $\mathrm{X}$-axis, while the $\mathrm{Y}$-axis shows the output voltage at the topmost nanowire. Red bars correspond to true outputs, blue bars denote false outputs.

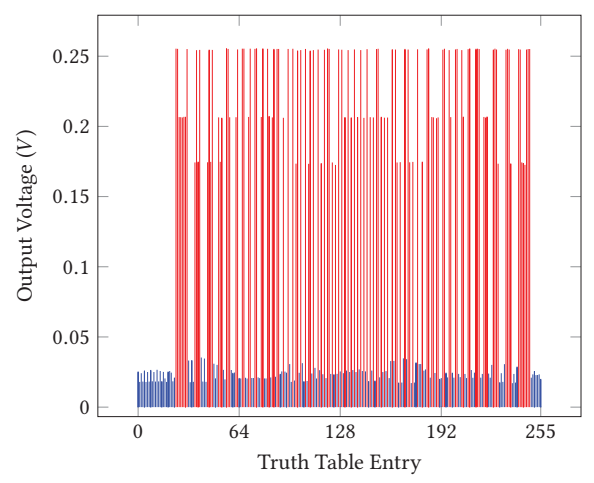

Figure 5: Output of a $24 \times 24$ for the fourth-output-bit of a multiplier. The $\mathrm{X}$-axis represents the truth table entry, and the Y-axis represents the voltage of the output nanowire. Red bars correspond to output-voltage for true outputs, blue bars denote voltages for $f$ alse outputs. The maximum voltage for a false output is $35 \mathrm{mV}$ while the minimum voltage for a true output is $172 \mathrm{mV}$. Gap of $137 \mathrm{mV}$ is almost 4 times the maximum voltage of the false output. 
Table 2: Comparison of the previous FBDD based approach [17] with the current approach for the outputs of a 4-bit multiplier

\begin{tabular}{llll}
\hline \multirow{2}{*}{ Bit Index } & \multicolumn{2}{l}{ FBDD based Crossbar Synthesis using } & \\
\cline { 2 - 3 } & $\begin{array}{l}\text { Most-frequent- } \\
\text { variable in DNF }\end{array}$ & Current Approach & Improvement \\
\hline 1 (LSB) & 2 by 2 & 2 by 2 & $0 \%$ \\
2 & 4 by 5 & 4 by 5 & $0 \%$ \\
3 & 8 by 7 & 10 by 9 & $-60.7 \%$ \\
4 & 35 by 34 & 24 by 24 & $51.6 \%$ \\
5 & 47 by 49 & 49 by 50 & $-6.4 \%$ \\
6 & 47 by 45 & 39 by 38 & $29.9 \%$ \\
7 & 28 by 28 & 22 by 24 & $32.7 \%$ \\
8 (MSB) & 10 by 11 & 10 by 11 & $0 \%$ \\
\hline Total & Area $=6582$ & Area $=5260$ & $20.1 \%$ \\
\hline
\end{tabular}

Table 3: Comparison of the previous FBDD based approach [17] with the current approach for different RevLib [25] benchmarks

\begin{tabular}{llll}
\hline \multirow{4}{*}{ Bit Index } & \multicolumn{2}{c}{ Crossbar Synthesis using } & \\
\cline { 2 - 3 } & $\begin{array}{l}\text { Most-frequent- } \\
\text { variable in DNF }\end{array}$ & Current Approach & Improvement \\
\hline 4gt4_20 & 3 by 4 & 3 by 3 & $25 \%$ \\
4gt4_22 & 2 by 3 & 2 by 3 & $0 \%$ \\
4 mod5_8 & 3 by 4 & 3 by 5 & $-25 \%$ \\
alu_9 & 5 by 3 & 5 by 3 & $0 \%$ \\
cm152a_130 & 6 by 6 & 5 by 8 & $-11.1 \%$ \\
ex1_150 & 6 by 5 & 6 by 5 & $0 \%$ \\
ex1_151 & 10 by 9 & 9 by 6 & $40 \%$ \\
ex1_152 & 7 by 7 & 6 by 6 & $26 \%$ \\
sym6_663 & 16 by 16 & 16 by 19 & $-18.8 \%$ \\
\hline
\end{tabular}

\section{CONCLUSION}

In this work, we have synthesized compact crossbars for in-memory computing of Boolean functions. We have used free binary decision diagrams to control and manage the sneak paths in a synthesized crossbar. Unlike ROBDDs, FBDDs do not have the requirement of a fixed global variable ordering which increases their representational power. But it also opens the question of how to find optimal variable orderings. The novelty of this work lies in the use of circuitsize for finding better variable orderings in FBDDs which result in compact crossbars. Our approach yields upto $50.1 \%$ smaller crossbars than the previous FBDD-based approach [17]. Overall, our approach has reduced the area-requirement for the 4-bit multiplier by $20.1 \%$. Besides the multiplier, we have also demonstrated the generality of our approach by synthesizing crossbars for other RevLib benchmarks. Our approach is also not memristor specific. It can be used for in-memory computing with other Resistive Ram devices.

\section{ACKNOWLEDGEMENT}

The authors would like to thank the US Air Force for support provided through the AFOSR Young Investigator Award to Sumit Jha. The authors acknowledge support from the National Science Foundation Software \& Hardware Foundations \#1438989 and Exploiting Parallelism \& Scalability \#1422257 projects. This material is based upon work supported by the Air Force Office of Scientific Research under award number FA9550-16-1-0255. The authors would also like to thank Fulbright U.S. Student Program for providing doctoral fellowship to Amad Ul Hassen.

\section{REFERENCES}

[1] M Mitchell Waldrop. The chips are down for Moore's law. Nature, 530(7589):144$147,2016$.

[2] John Von Neumann. First Draft of a Report on the EDVAC. IEEE Annals of the History of Computing, 15(4):27-75, 1993.

[3] Muhammad Shafique, Siddharth Garg, Jörg Henkel, and Diana Marculescu. The EDA Challenges in the Dark Silicon Era: Temperature, Reliability, and Variability Perspectives. In Proceedings of the 51st Annual Design Automation Conference, DAC '14, pages 1-6, New York, NY, USA, 2014. ACM.

[4] Luis Ceze, Mark D Hill, and Thomas F Wenisch. Arch2030: A Vision of Computer Architecture Research over the Next 15 Years. arXiv preprint arXiv:1612.03182, 2016.

[5] H-S Philip Wong and Sayeef Salahuddin. Memory leads the way to better computing. Nature Nanotechnology, 10(3):191-194, 2015.

[6] A. Bricalli, E. Ambrosi, M. Laudato, M. Maestro, R. Rodriguez, and D. Ielmini. Resistive Switching Device Technology Based on Silicon Oxide for Improved ON-OFF Ratio-Part II: Select Devices. IEEE Transactions on Electron Devices, 65(1):122-128, Jan 2018

[7] Dmitri B Strukov, Gregory S Snider, Duncan R Stewart, and R Stanley Williams. The missing memristor found. Nature, 453(7191):80-83, May 2008.

[8] Julien Borghetti, Gregory S Snider, Philip J Kuekes, J Joshua Yang, Duncan R Stewart, and R Stanley Williams. 'Memristive' switches enable 'stateful' logic operations via material implication. Nature, 464(7290):873-876, 2010.

[9] Eero Lehtonen, JH Poikonen, and Mika Laiho. Two memristors suffice to compute all Boolean functions. Electronics letters, 46(3):239-240, 2010.

[10] Shahar Kvatinsky, Guy Satat, Nimrod Wald, E.G. Friedman, Avinoam Kolodny, and Uri C. Weiser. Memristor-Based Material Implication (IMPLY) Logic: Design Principles and Methodologies. IEEE Transactions on Very Large Scale Integration (VLSI) Systems, 22(10):2054-2066, Oct 2014

[11] C. E. Shannon. A symbolic analysis of relay and switching circuits. Transactions of the American Institute of Electrical Engineers, 57(12):713-723, Dec 1938.

[12] S. Kvatinsky, D. Belousov, S. Liman, G. Satat, N. Wald, E. G. Friedman, A. Kolodny, and U. C. Weiser. MAGIC-Memristor-Aided Logic. IEEE Transactions on Circuits and Systems II: Express Briefs, 61(11):895-899, Nov 2014.

[13] Sumit Kumar Jha, Dilia E Rodriguez, Joseph E Van Nostrand, and Alvaro Velasquez. Computation of boolean formulas using sneak paths in crossbar computing, April 19 2016. US Patent 9,319,047.

[14] Zahiruddin Alamgir, Karsten Beckmann, Nathaniel Cady, Alvaro Velasquez, and Sumit Kumar Jha. Flow-based Computing on Nanoscale Crossbars: Design and Implementation of Full Adders. In Circuits and Systems (ISCAS), 2016 IEEE International Symposium on, pages 1870-1873. IEEE, 2016.

[15] Dwaipayan Chakraborty and Sumit Kumar Jha. Automated synthesis of compact crossbars for sneak-path based in-memory computing. In Design, Automation Test in Europe Conference Exhibition (DATE), 2017, pages 770-775. IEEE, March 2017.

[16] A. U. Hassen. Automated synthesis of compact multiplier circuits for in-memory computing using ROBDDs. In 2017 IEEE/ACM International Symposium on Nanoscale Architectures (NANOARCH), pages 141-146, July 2017.

[17] A. Ul Hassen, D. Chakraborty, and S. K. Jha. Free Binary Decision Diagram-Based Synthesis of Compact Crossbars for In-Memory Computing. IEEE Transactions on Circuits and Systems II: Express Briefs, 65(5):622-626, May 2018.

[18] Yuval Cassuto, Shahar Kvatinsky, and Eitan Yaakobi. Information-Theoretic Sneak-Path Mitigation in Memristor Crossbar Arrays. IEEE Transactions on Information Theory, 62(9):4801-4813, Sept 2016.

[19] Chang Yeong Lee. Representation of switching circuits by binary-decision programs. The Bell System Technical fournal, 38(4):985-999, July 1959.

[20] Sheldon B. Akers. Binary Decision Diagrams. IEEE Transactions on Computers, C-27(6):509-516, June 1978.

[21] Randal E. Bryant. Graph-Based Algorithms for Boolean Function Manipulation. IEEE Transactions on Computers, C-35(8):677-691, Aug 1986.

[22] Ingo Wegener. Branching Programs and Binary Decision Diagrams: Theory and Applications, volume 4 of Monographs on Discrete Mathematics and Applications. SIAM, 2000.

[23] M. Fujita, H. Fujisawa, and N. Kawato. Evaluation and improvement of Boolean comparison method based on binary decision diagrams. In [1988] IEEE International Conference on Computer-Aided Design (ICCAD-89) Digest of Technical Papers, pages 2-5, Nov 1988.

[24] Fadi A Aloul, Igor L Markov, and Karem A Sakallah. Efficient Gate and Input Ordering for Circuit-to-BDD Conversion. In IWLS, pages 137-142, 2002. 
[25] R. Wille, D. Große, L. Teuber, G. W. Dueck, and R. Drechsler. RevLib: An Online Resource for Reversible Functions and Reversible Circuits. In Int'l Symp. on MultiValued Logic, pages 220-225, 2008. RevLib is available at http://www.revlib.org. 